\title{
Deep CCD Surface Photometry of Galaxy Clusters I: Methods and Initial Studies of Intracluster Starlight
}

\author{
John J. Feldmeier ${ }^{1}$, J. Christopher Mihos ${ }^{2}$, Heather L. Morrison ${ }^{1,2}$, Steven A. Rodney, Paul \\ Harding \\ johnf@eor.astr.cwru.edu, hos@burro.astr.cwru.edu, \\ heather@vegemite.astr.cwru.edu, sar9@smaug.astr.cwru.edu, \\ harding@billabong. astr.cwru.edu \\ Department of Astronomy, Case Western Reserve University, 10900 Euclid Ave, \\ Cleveland, $\mathrm{OH} 44106$
}

\begin{abstract}
We report the initial results of a deep imaging survey of galaxy clusters. The primary goals of this survey are to quantify the amount of intracluster light as a function of cluster properties, and to quantify the frequency of tidal debris. We outline the techniques needed to perform such a survey, and we report findings for the first two galaxy clusters in the survey: Abell 1413, and MKW 7. These clusters vary greatly in richness and structure. We show that our surface photometry reliably reaches to a surface brightness of $\mu_{\mathrm{V}}=26.5 \mathrm{mag} \operatorname{arsec}^{-2}$. We find that both clusters show clear excesses over a best-fitting $r^{1 / 4}$ profile: this was expected for Abell 1413, but not for MKW 7. Both clusters also show evidence of tidal debris in the form of plumes and arc-like structures, but no long tidal arcs were detected. We also find that the central cD galaxy in Abell 1413 is flattened at large radii, with an ellipticity of $\approx 0.8$, the largest measured ellipticity of any cD galaxy to date.
\end{abstract}

Subject headings: galaxies: clusters: general — galaxies: clusters: individual (Abell 1413, MKW 7) — galaxies: interactions - galaxies: kinematics and dynamics

\footnotetext{
${ }^{1}$ Visiting Astronomer, Kitt Peak National Observatory, National Optical Astronomy Observatory, which is operated by the Association of Universities for Research in Astronomy, Inc. (AURA) under cooperative agreement with the National Science Foundation.

${ }^{2}$ Cottrell Scholar of Research Corporation and NSF CAREER fellow; Also Department of Physics, Case Western Reserve University.
} 


\section{Introduction}

The concept of intracluster starlight was first proposed by Zwicky (1951), who claimed to detect excess light between the galaxies of the Coma cluster. Follow-up photographic searches for intracluster luminosity in Coma and other rich clusters (e.g., Welch \& Sastry 1971; Melnick, White \& Hoessel 1977, see Víchez-Gómez 1999 \& Feldmeier 2000 for reviews) produced mixed results, and it was not until the advent of CCDs that more precise estimates of the amount of intracluster starlight were made (e.g., Uson, Boughn, \& Kuhn 1991; Vílchez-Gómez, Pelló \& Sanahuja 1994; Bernstein et al. 1995; Gonzalez et al. 2000). These observations are extremely difficult to perform and interpret due to the low surface brightness

of the phenomenon: typically, the surface brightness of intracluster light is less than $1 \%$ of the brightness of the night sky. Measurements of this luminosity must also contend with the problems presented by scattered light from nearby bright objects and the contribution of discrete sources.

Despite these difficulties, intracluster light (ICL) is of potentially great interest to studies of galaxy and galaxy cluster evolution. The dynamical evolution of cluster galaxies is complex, involving poorly understood processes such as galactic encounters, dark matter, cluster accretion, and tidal stripping (cf. Dressler 1984). The ICL provides a direct way to study these different mechanisms. Various studies have suggested that anywhere between $10 \%$ and $70 \%$ of a cluster's total luminosity may be contained in the ICL (Richstone \& Malumuth 1983; Miller 1983), with a strong dependence on the dynamical state of the cluster. The properties of the ICL may also be sensitive to the distribution of dark matter in cluster galaxies. Simulations have shown that the structure of dark matter halos in galaxies plays a central role in the formation and evolution of tidal debris (Dubinski, Mihos, \& Hernquist 1996, 1999). If cluster galaxy dark halos are tidally truncated at small radii (e.g., Whitmore, Forbes, \& Rubin 1988), stripped material can be more easily unbound from the galaxies and end up being distributed smoothly throughout the cluster. Conversely, if cluster galaxy halos survive, some tidally stripped material may remain bound to these galaxies, leaving them embedded in very low surface brightness "cocoons." The ICL may act as a sensitive probe of the mechanics of tidal stripping, the distribution of dark matter around galaxies, and cluster evolution in general.

Recently, much progress has been made in the study of intracluster starlight on numerous fronts. Individual intracluster stars, namely planetary nebulae detected from the ground and red giants detected using HST, have been discovered in the Virgo and Fornax clusters (Arnaboldi, et al. 1996; Theuns \& Warren 1997; Mendez et al. 1997; Ciardullo et al. 1998; Feldmeier, Ciardullo, \& Jacoby 1998; Ferguson, Tanvir, \& von Hippel 1998; Feldmeier 2000; Durrell, et al. 2002). Although some of the intracluster planetary candidates were later found 
to be background objects (Kudritzki et al. 2000; Ciardullo et al. 2002), most are bona-fide intracluster planetary nebulae (Freeman et al. 2000; Ciardullo et al. 2002). There is also some evidence for intracluster supernovae, though the results here are more tentative, and the rate may be significantly smaller than that seen in galaxies (Smith 1981; Gal-Yam \& Maoz 2000; Tyson et al. 2002). These individual intracluster stars give the promise of studying in detail the kinematics, metallicity, and age of the intracluster stellar population in nearby galaxy clusters.

Another area of progress is the advent of modern numerical studies of the dynamical evolution of galaxies in clusters. High-resolution N-body simulations now have the ability to follow hundreds of cluster galaxies interacting within a cosmological context (Moore et al. 1996; Dubinski 1998). These high-quality simulations finally allow testable predictions of the production and properties of intracluster starlight (Moore, Lake, \& Katz 1998; Dubinski, Murali, \& Ouyed 2000; Korchagin, Tsuchiya, \& Miyama 2001). When combined with earlier theoretical studies, (Gallagher \& Ostriker 1972; Merritt 1983; Richstone \& Malumuth 1983; Miller 1983; Merritt 1984) there is now a growing theoretical framework in which to interpret observations of the ICL.

Recently, another aspect of intracluster starlight has been discovered: tidal debris arcs. These features are large $(\sim 100 \mathrm{kpc})$, low-surface brightness $\left(\mu_{\mathrm{V}} \sim 26 \mathrm{mag} \operatorname{arsec}^{-2}\right)$ arc-like structures found in nearby galaxy clusters, and are not due to gravitational lensing. These arcs have been found in the Coma and Centaurus clusters (Trentham \& Mobasher 1998; Gregg \& West 1998; Calcańeo-Roldán, et al. 2000). It has been proposed that these arcs are due to tidal interactions between galaxies and the cluster's gravitational potential (Moore et al. 1996). Since several of these debris arcs were found by chance, it is plausible that they might be present in other galaxy clusters. The scientific potential for these arcs is exciting. By observing the morphology, and - in the future - kinematics of these stellar streams, much can be learned about the orbits of the infalling galaxies, and the gravitational potential of the galaxy cluster (i.e., Calcańeo-Roldán, et al. 2000).

Although the presence of intracluster stars has been clearly demonstrated, there is little information on how the amount and distribution of intracluster starlight varies with the properties of the cluster it inhabits. We do not yet have a global picture of the prevalence of the ICL in galaxy clusters, nor of the information it contains about the dynamical state of clusters, both of which are crucial in developing more advanced models of cluster evolution. Studies of individual intracluster stars are invaluable in nearby clusters, but are flux-limited and so cannot probe the evolution of intracluster light to higher redshift. Finally, there is little data on how common tidal debris arcs might be in galaxy clusters. Currently, the majority of tidal debris arcs discovered have been found in the Coma cluster (Trentham \& 
Mobasher 1998; Gregg \& West 1998; Calcańeo-Roldán, et al. 2000). The Coma cluster is well known to be unusually rich (c.f., Dressler 1984), and it is possible that it might contain an unusually large number of tidal debris arcs.

Finally, another interesting facet of the ICL in clusters is the nature of $\mathrm{cD}$ envelopes. First identified in deep photographic imaging of clusters, $\mathrm{cD}$ galaxies are characterized by an excess of diffuse light (compared to an $r^{1 / 4}$ law) at large radius. The origin of $\mathrm{cD}$ envelopes remains unclear: are they formed in the initial stages of cluster collapse, or later, as galaxies continue to fall in the cluster and become tidally stripped? The detailed light distribution of cD envelopes may hold clues to the answer. The statistical mechanics of violent relaxation naturally produces $r^{1 / 4}$-like profiles (Lynden-Bell 1966); if cD envelopes form during cluster collapse, they should show such a profile. On the other hand, continued accretion and stripping of infalling galaxies need not produce an $r^{1 / 4}$ profile, as the distribution of stripped light will be more sensitive to the orbital energy and angular momentum of the infalling galaxies. While the characterization of $\mathrm{cD}$ envelopes as an excess of light over an $r^{1 / 4}$ profile would seem to argue for a stripping origin, most studies of $\mathrm{cD}$ galaxies have used older photographic data, with very uncertain flat fielding characteristics. Newer work using CCD imaging has shown at least one case where a $\mathrm{cD}$ galaxy may in fact be well characterized by a pure $r^{1 / 4}$ law (Gonzalez et al. 2000). In light of this result, revisiting the question of the structure of $\mathrm{cD}$ galaxies using deep CCD imaging may shed new light on the origin of the $\mathrm{cD}$ envelopes and the evolution of galaxy clusters.

To address these questions, we have begun deep imaging of a sample of galaxy clusters to quantify the structure of ICL. Although quantitative surface photometry several magnitudes below sky is an extremely challenging task, over the past decade the necessary CCD imaging techniques have been developed and carried out on both galaxies (e.g., Morrison, Boroson, \& Harding 1994; Morrison et al. 1997; Fry et al. 1999; Zheng et al. 1999) and galaxy clusters (e.g., Tyson, Kochanski \& dell'Antonio 1998; Gonzalez et al. 2000). Using these techniques, our plan is to image galaxy clusters which differ in richness, concentration, and sub-structure to quantify how the ICL changes as a function of environment. In conjunction with increasingly sophisticated models of cluster galaxy evolution, such observations can provide constraints on the evolution of clusters and cluster galaxies, the formation of the ICL, and the distribution of dark matter in cluster galaxies. In this paper, we describe in detail our imaging techniques, and show results from the first two clusters surveyed. 


\section{Selection Criteria}

Our program is aimed at studying the ICL in clusters possessing a variety of structural properties, in order to probe the relationship between the ICL and cluster environment. Our initial sample will primarily focus on Abell (Abell, Corwin, \& Olowin 1989) clusters of distance class $5-6(\mathrm{z} \approx 0.1-0.175)$ with differing richness and Bautz-Morgan classifications. The lower end of the redshift range is chosen such that the inner $\sim 0.75 \mathrm{Mpc}$ of the cluster fits on the field-of-view of moderate size CCD detectors, allowing us to study the cluster as a whole without mosaicing, and permitting a reasonable amount of sky at the outer edge of the field for sky subtraction. The upper limit is set so that $(1+z)^{4}$ surface brightness dimming is not prohibitive, and also to prevent the angular size of any tidal arcs from being too small. For comparison purposes, we also observe nearby poor galaxy clusters from the MKW/AWM catalogs of poor clusters that appear to contain cD galaxies (Morgan, Kayser, \& White 1975; Albert, White, \& Morgan 1977). These clusters will provide a significantly different cluster environment in terms of density and dynamical interaction.

Scattered starlight is a crucial source of systematic error in our program. Therefore, we must screen our candidate clusters carefully, making sure there are no bright stars in the CCD field or up to several degrees nearby. Because of the complex spatial nature of the scattered light distribution, we do our screening by manual inspection of the original

Palomar Sky Survey (POSS) plates in the area around the target cluster. Approximately half of our candidate clusters are rejected by this process. Once the cluster passes both the catalog criteria and scattered light tests, it is included as a potential target.

\section{Cluster Properties}

For the first targets of our survey, we chose two galaxy clusters with greatly differing

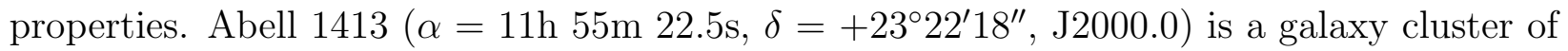
richness class 3 (richer than $95 \%$ of the original Abell catalog), with a Bautz-Morgan type of I (cD dominated; Leir \& van den Bergh 1977) and a Rood-Sastry type of cD (Struble \& Rood 1987). Its central cD galaxy has been studied with photographic surface photometry at large radii, and CCD surface photometry at smaller radii (Oemler 1976; Schombert 1986, 1988; Schneider, Gunn, \& Hoessel 1983; Porter, Schneider, \& Hoessel 1991) numerous times, allowing us to compare our results with others. These earlier studies imply that the properties of A1413's cD halo are extremely impressive: Oemler (1976) found that the cD halo of Abell 1413 might extend as far as 24' $\left(2.4 h^{-1} \mathrm{Mpc} ; \mathrm{H}_{0}=100 h \mathrm{~km} \mathrm{~s}^{-1} \mathrm{Mpc}^{-1}\right)$ away from the center of the cluster, and Morgan \& Lesh (1965) indicated that Abell 1413 might be the largest of all cD galaxies. Schombert (1988) studied Abell 1413 in detail, and found that the 
$\mathrm{cD}$ halo extended to $\sim 500 \mathrm{kpc} \mathrm{h}^{-1}$, with a large excess over the best-fitting de Vaucouleurs $\mathrm{r}^{1 / 4}$ profile of the inner regions. Abell 1413 has a relatively high X-ray temperature $(8 \mathrm{keV}$; White 2000), and has a Sunyaev-Zeldovich decrement (Grainge et al. 1996), confirming that the cluster is indeed massive. Therefore, Abell 1413 is a representative example of a rich cluster, albeit containing an abnormally large cD galaxy.

MKW 7 (WBL 514 - White et al. (1999); $\alpha=14 \mathrm{~h} 34 \mathrm{~m} 00.9 \mathrm{~s}, \delta=+03^{\circ} 46^{\prime} 52^{\prime \prime}$, J2000.0) is a poor galaxy cluster whose brightest cluster galaxy was found to be cD-like in appearance from inspection of the Palomar Sky Survey (Morgan, Kayser, \& White 1975, see Tonry 1987; Schombert 1992 for discussions of cD classification). It has a richness class of -1 (Bahcall 1980), and in galaxy counts, is over a factor of eight poorer than Abell 1413. Photographic surface photometry has been made of MKW 7's brightest cluster galaxy (Morbey \& Morris 1983), but these measurements are complicated by the presence of a $m_{\mathrm{V}} \approx 11.9$ star within $21^{\prime \prime}$ of the galaxy nucleus. Van den Bergh (1977) notes that the brightest cluster galaxy is embedded in a bright but asymmetrical envelope. From galaxy density maps and redshift information Beers et al. (1995) argue that MKW 7 is gravitationally bound to another poor cluster, MKW 8, which is within $1.5 \mathrm{~h}^{-1} \mathrm{Mpc}$. MKW 7 has been detected in X-rays multiple times (e.g., Price et al. 1991), but no gas temperature has yet been determined. In comparison to Abell 1413, MKW 7 is a poorer, less dynamically evolved cluster.

\section{A Note on nomenclature}

We note that the term "intracluster starlight" has been applied in many different ways in the literature. A dynamical definition might be stars which are unbound from any individual cluster galaxy, yet bound to the cluster as a whole. From an observational point of view, of course, this definition is inaccessible without knowing the detailed kinematics of the ICL and the total mass distribution in the cluster. With deep imaging, the definition of ICL can only be made based on the surface brightness distribution within the cluster. Indeed, it is debatable whether cD envelopes should be considered as ICL - is the envelope a feature of the $\mathrm{cD}$ galaxy itself, or are both the $\mathrm{cD}$ and the envelope simply material which has collected at the bottom of the cluster potential well? Uson, Boughn \& Kuhn (1991) succinctly summarize the situation in their observations of Abell 2029:

"...Whether this diffuse light is called the $\mathrm{cD}$ envelope or diffuse intergalactic light is a matter of semantics; it is a diffuse component which is distributed with elliptical symmetry about the center of the cluster potential..." 
Based on surface photometry alone it is difficult to disentangle luminosity associated with a $\mathrm{cD}$ envelope from that of the extended ICL, and in fact such a distinction may not be well motivated from a physical point of view. For the purposes of our work, we will simply use the term "intracluster light" to refer to the diffuse light in galaxy clusters, and address issues related to $\mathrm{cD}$ envelopes, diffuse arcs, and extended ICL in the context of diffuse light as a whole.

\section{Observations and Reductions}

\subsection{Observations}

The data for Abell 1413 and MKW 7 were obtained over two photometric nights during a four night run in 2000 April, using the $2.1 \mathrm{~m}$ telescope at Kitt Peak National Observatory ${ }^{3}$. The images were taken using a 2048 x 2048 Tektronix CCD (T2KA). With this setup, the field of view is $10.4 \mathrm{arcmin}^{2}$, with each pixel imaging $0.305^{\prime \prime}$ of sky. The gain was set at the default value of $3.6 \mathrm{e}^{-} \mathrm{ADU}^{-1}$ and the readout noise was $4 \mathrm{e}^{-}$(1.1 ADU). All exposures were made through a Washington $M$ filter, which is similar to Johnson $V$ but is slightly bluer in mean wavelength and therefore contains fewer airglow emission lines (see Figure 1). These airglow lines, produced in the upper atmosphere by a variety of mechanisms (Roach \& Gordon 1973), are a significant source of sky background, and are well known to be variable over timescales of minutes (e.g., Pilachowski et al. 1989; Krisciunas 1997; Morrison et al. 1997). Therefore, to reduce the sky background, and to simplify the process of sky subtraction and flat-fielding, we chose the Washington $M$ filter for our observations. We transformed these observations to Johnson $V(\S 5.4)$, and unless otherwise stated, all surface brightnesses are given in $V$ magnitudes.

We began the telescope run by preparing the detector, telescope and filter to reduce the amount of scattered light, which sets the ultimate limit to our surface photometry. We first placed a black cardboard mask over the detector's dewar window in order to reduce scattered light from the mounting hardware surrounding the CCD. These metallic components are highly reflective, and a clear difference in the amount of scattered light is readily apparent. We next took pin-hole images of the telescope pupil to search for other sources of scattered light (Grundahl \& Sørensen 1996), and baffled any such areas with black cloth.

An accurate flat-field is critical to the success of our program. As mentioned in $\S 1$,

\footnotetext{
${ }^{3}$ Kitt Peak National Observatory is a division of NOAO, which is operated by AURA, under cooperative agreement with the National Science Foundation.
} 
we are interested in recovering a signal that is less than $1 \%$ of the sky background. Our flat field must be at least a few times more accurate than this $1 \%$ value for our data to be meaningful. For this reason, dome flat fields cannot be used due to possible scattered light, differing pupil illuminations, and intrinsic color differences. For similar reasons, twilight flats are also inadequate for our purposes. Therefore, dark sky flats are a neccesity, and we performed the observations in the manner described by Morrison et al. (1997). Half of the time was used observing the galaxy clusters, and the other half was used to obtain dark sky flats. The dark sky flat images were taken at pre-determined areas away from bright stars at approximately the same hour angle and declination as the cluster images. Over the course of the observing run, a total of nine images were taken of Abell 1413, 12 images were taken of MKW 7, and 23 dark sky flats were obtained. For each of the cluster and sky images, the exposure time was $900 \mathrm{~s}$.

\subsection{Correction for non-linearity:}

After our run, we were made aware of the presence of non-linearity in the T2KA detector by K. Stanek (reported in Mochejska et al. 2001). Figure 2 shows the comparison of stellar photometry derived from a 60s test exposure of MKW 7 and a median-combined exposure of $900 \mathrm{~s}$, both reduced in the standard manner. Although the scatter is large, a clear nonlinearity is present in the data. We fit the residuals in magnitude with a least-squares linear model, and found a residual slope of $0.0095 \pm 0.0008$ magnitudes per magnitude. This is consistent with the measurements of Mochejska et al. (2001) for their test photometry of NGC 7789 (see their figure 3). Since the non-linear behavior is identical to that seen in the data presented in Mochejska et al. (2001), the data was taken with the same instrument only 5-6 months apart, and the Mochesjska et al. data better constrains the effect, we adopt identical corrections for non-linearity:

$$
I_{e}=I_{i} \cdot\left(c_{1}+c_{2} \cdot \frac{I_{i}}{32767}+c_{3} \cdot\left(\frac{I_{i}}{32767}\right)^{2}\right)
$$

where $I_{i}$ is the measured intensity, and $I_{e}$ is the corrected intensity in ADU. The constants derived by Mochejska et al. (2001) for a gain of $3.6 \mathrm{e}^{-} \mathrm{ADU}^{-1}$ are:

$$
c_{1}=0.983282, c_{2}=-0.0765595, c_{3}=0.0252555
$$


Following Mochejska et al. (2001), we used the IRLINCOR task within $\operatorname{IRAF}^{4}$, to correct the data for non-linearity after overscan removal and bias subtraction.

For two reasons, the non-linearity is less of a problem for our project than it might first seem. First, since our sky flats have exactly the same exposure time as our data, they have the identical non-linearity inherent in the exposures, so any difficulty in flat-fielding is canceled out to first order. Second, at low surface brightness levels, the error caused by any nonlinearity is significant, but relatively small. Figure 3 shows the difference in magnitudes between the corrected, and non-corrected sky-subtracted surface brightness. Nonetheless, any error in the correction for non-linearity adds a source of error to our surface brightness estimates. To determine the amount of such error, we obtained the linearity data from Mochejska et al. (2001) (kindly provided to us by B. Mochejska), and replicated the linearity fit. We found the identical constants with the following $1-\sigma$ errors on the parameters:

$$
\sigma_{c_{1}}=0.0052, \sigma_{c_{2}}=0.012, \sigma_{c_{3}}=0.0057
$$

This uncertainty is added to our final error model (see $\S 6.4$ ).

\subsection{Flat Fielding}

After overscan removal and bias subtraction (done in the standard manner), we constructed a "master" sky flat from the dark sky images taken. First, each individual sky flat was visually inspected to ensure that no bright stars or scattered light patterns were present in the image. This is necessary because in some of our exposures of candidate galaxy clusters at the telescope, we found that a grid-like scattered light pattern appeared. This pattern was rotated 45 degrees from the CCD axes, and typically covered an area of 370 by 350 pixels. When the pattern did appear, its surface brightness varied, but it could be as large as $\mu_{\mathrm{V}} \approx 25.2 \mathrm{mag} \operatorname{arsec}^{-2}$. Three of the dark-sky images were found to have unacceptably large scattered light patterns, and were removed from the list, leaving 20 dark-sky images to construct the dark sky flat. Three other dark sky images also contain the scattered light pattern, but the amplitude of the pattern was so small in these cases (their presence was barely visible on the image) that they were left in the sample. No scattered light patterns were seen in any image of Abell 1413 and MKW 7.

To construct the best possible master sky flat, we combined the individual sky frames

\footnotetext{
${ }^{4}$ IRAF is distributed by the National Optical Astronomy Observatories, which are operated by the Association of Universities for Research in Astronomy, Inc., under cooperative agreement with the National Science Foundation.
} 
using a very accurate determination of their modes. We do this using the iterative procedure described in Morrison et al. (1997). We first pre-scaled the images by their mode, found from the IMSTAT task within IRAF. Prescaling is important because even with our relatively narrow $M$ filter, the modal value of the sky images varies by up to $22 \%$, from maximum to minimum. Then we combined the individual, modal-divided sky images to make a preliminary flat-field frame, using IRAF's IMCOMBINE task, with the CCDCLIP algorithm, set to remove pixels which differed from the median by more than $2 \sigma$.

Each of the individual sky frames were then divided by this preliminary flat-field frame to reduce the width of the distribution of modal sky values, making rejection of outliers due to faint stars and stellar wings more accurate. The flat-fielded sky frames were then averaged into 50 by 50 pixel bins, and a plane was then fit to the binned-up images using the IRAF task IMSURFIT. This step is necessary because each individual sky frame has noticeable sky variations across the image due to a number of atmospheric effects such as airglow (Roach \& Gordon 1973; Wild 1997; Zheng et al. 1999). Figure 4 shows the binned-up images for each sky frame after they have been flat-fielded. Clear systematic sky variations can be easily seen in the data. After the individual planes were fit and normalized, the sky frames were each divided by their normalized plane. The modes were then recalculated using our own software, and the entire procedure was repeated using the improved flat-field frame. The procedure was repeated until the calculated modes had converged (about 15 times in this case).

The galaxy cluster images were then flat-fielded by this final flat, and were registered using stars common to all frames and the IRAF tasks GEOMAP and GEOTRANS, using a 2nd order polynomial fit. A preliminary sky value was found for each cluster image by finding the mode of two regions on each chip well away from the center of the cluster, and averaging the results. This sky value was then subtracted from each image. The median sky value for Abell 1413 was 886.0 ADU pixel ${ }^{-1}$ and 932.7 $\mathrm{ADU}_{\text {pixel }}{ }^{-1}$ for MKW 7. After applying the photometric zero point in ( 55.4$)$ below, these correspond to $\mu_{\mathrm{V}}=21.11 \mathrm{mag} \mathrm{arsec}^{-2}$ and $\mu_{\mathrm{V}}=21.05 \mathrm{mag} \operatorname{arsec}^{-2}$, respectively. Since the source of sky brightness is mostly within the earth's atmosphere, we remove our 0.17 mag airmass $^{-1}$ extinction correction, and find that the average brightness of the night sky at zenith was approximately $\mu_{\mathrm{V}}=21.25 \mathrm{mag} \mathrm{arsec}^{-2}$, in reasonable agreement with the solar maximum value of $21.287 \pm 0.048$ of Krisciunas (1997).

With the overscan, bias-subtraction, flat-fielding and sky subtraction complete, we then combined the images together, using a $2 \sigma$ clipped median as before, and scaling for airmass. The final images for Abell 1413 and MKW 7 are displayed in Figures 5 and 6. The measured seeing (full-width at half-maximum) for the final combined images was 1". 22 for Abell 1413 and 1". 37 for MKW 7. 


\subsection{Photometric Zero Point:}

The Landolt star fields SA 98, SA 107, \& SA 110 (Landolt 1992) were observed, giving us a total of 37 well-observed standard stars over a range of color and airmass. For the purposes of our analysis, we converted our Washington $M$ exposures to $V$ band magnitudes. This transformation is straightforward because all of the Washington $M$ standard stars used in these observations are also Landolt (1992) $V$ standards. A photometric zero point of $\mathrm{V}$ $=21.09 \pm 0.04 \mathrm{mag} \operatorname{arcsec}^{-2}$ (corresponding to $1 \mathrm{ADU} \mathrm{s}^{-1}$ pixel $^{-1}$, and assuming a (B-V) color of 1.0 ) was determined. For a $900 \mathrm{~s}$ exposure this yields $\mathrm{V}=28.48 \mathrm{mag} \mathrm{arcsec}^{-2}$ corresponding to $1 \mathrm{ADU}$ per pixel at unit airmass. As our exposures were only taken in one filter, and we do not know the exact color of the intracluster light, we cannot add a color correction term to our target photometry, but from the standard star observations, we estimate its magnitude as less than $0.1 \mathrm{mag}$, over the entire likely color range of our target objects $(0.8 \leq(\mathrm{B}-\mathrm{V}) \leq 1.3)$. The color term is reasonably well fit as a linear function of $(\mathrm{B}-\mathrm{V})$, with a slope of 0.2 magnitudes per magnitude of $(\mathrm{B}-\mathrm{V})$ color.

\section{Analysis and Results}

We adopt an approximate angular size distance to Abell 1413 and MKW 7 of 465 and $111 \mathrm{Mpc}$ respectively, assuming redshifts of $\mathrm{z}=0.1427$ for Abell 1413 (Struble \& Rood 1999)

and $\mathrm{z}=0.0290$ for MKW 7 (Beers et al. 1995), a Hubble constant, $\mathrm{H}_{0}=75 \mathrm{~km} \mathrm{~s}^{-1} \mathrm{Mpc}^{-1}$, and a cosmology of $\Omega_{\mathrm{m}}=0.3, \Omega_{\Lambda}=0.7$. At these small redshifts, these distances depend little on $\Omega$. Given these assumed distances, 1 arcsecond subtends $\approx 2.3 \mathrm{kpc}$ in Abell 1413, and $\approx 0.52 \mathrm{kpc}$ in MKW 7 . The corresponding luminosity distance moduli, ignoring any K-corrections, are 39.0 for Abell 1413, and 35.4 for MKW 7.

\section{1. $\quad$ Masking}

In order to reach the faintest possible surface brightness levels of the $\mathrm{cD}$ galaxy + intracluster light, we must mask out all other sources - both stars and galaxies - in the frame. We begin by creating a binary mask image where one indicates a good pixel and zero indicates a bad pixel. This has the advantage of allowing us to visually compare our mask image at any point in the construction process by simply multiplying the mask by the data image, and displaying the results.

We first begin by masking out the stars in each image. Since we are concerned with very low surface brightness, we must determine the point spread function (PSF) out to very 
large radii. Using the DAOPHOT (Stetson 1987) package, we detected all of the stars in the frame down to a signal-to-noise of three, and used a subset of bright stars to first determine the PSF out to a radius of 20 pixels. We then used this preliminary PSF to mask out all of the stars and small galaxies around two bright saturated stars in our Abell 1413 data. Saturated stars have much higher signal-to-noise in the wings on the PSF, which are our primary concern. Other sources, such as resolved galaxies and stellar diffraction spikes, were removed manually. Then the unmasked pixels from the two saturated stars were averaged in radial annuli, and joined to the preliminary PSF (which measures the inner core of the star more accurately). The final radial profile is displayed in Figure 7. Using this large-radius PSF, and the list of stars found by DAOPHOT, we masked all stellar sources in the frame out to a radius where the magnitude-scaled PSF was 1 ADU above the sky value.

Next, we must mask out all of the galaxies in each cluster, excluding the central cD. Unresolved background galaxies have been treated as point sources, and have already been masked by the DAOPHOT procedure above, but many resolved sources remain in both clusters. We chose to mask out the galaxies using the segmentation image from the SExtractor software package (V2.2.1; Bertin \& Arnouts 1996). Again, since we must mask down to very low surface brightness levels, the SExtractor detection parameters are set for faint surface brightness levels. After experimentation, we adopted a minimum detection threshold of 4 pixels that were $0.6 \sigma$ above the local sky background. This corresponds to $3.3 \mathrm{ADU}$ in Abell 1413, and 2.7 ADU in MKW 7. Assuming Gaussian statistics, the probability of a false SExtractor detection at these low-light levels is $5.6 \times 10^{-3}$ per four pixel block. This is uncomfortably high, and allows for the possibility of "over-masking" our data, that is, masking out noise spikes, instead of real objects. This would alter the noise properties of our data, and lead to systematic errors in our surface photometry. However, we need the low threshold to ensure that the low surface brightness outer regions of large, luminous galaxies are being properly masked.

We deal with the "over-masking" problem by running SExtractor without de-blending the various detections, that is, not assigning faint isolated objects as part of a much brighter object. Then, we removed all sources whose total magnitude was fainter than a cut-off value. We found the cut-off value in two different ways. First, we created the raw galaxy brightness distribution for both clusters by selecting all objects with a stellarity index less than 0.5, where the stellarity index defines the likelihood that a source is or is not extended through measurements of image moments by SExtractor's neural network (Bertin \& Arnouts 1996). We then noted where the raw galaxy brightness distribution slope rapidly increased. This will indicate the onset of the noise spikes. Second, we ran SExtractor on the mathematical inverse of each cluster image:

$$
I_{\text {inverse }}(x, y)=-I_{\text {image }}(x, y)
$$


where $\mathrm{x}, \mathrm{y}$ are the pixel coordinates of the image, and $\mathrm{I}(\mathrm{x}, \mathrm{y})$ is the flux in ADU at each point. We then found the brightness distribution of negative noise spikes, which should provide an accurate measure of the cut-off value, assuming that the noise is symmetrically distributed. The results of both of these tests are displayed in Figure 8, and are in good agreement with one another. We set the cutoff magnitude to 23.8 for Abell 1413 and 23.0 for MKW 7, and removed all sources from the segmentation image that were below this value.

There is one other change that we must make to the segmentation image. After inspection of the corrected segmentation mask multiplied by the data, we occasionally found small groups of pixels that were completely surrounded by a large number of masked pixels. These "islands" of unmasked pixels are due to SExtractor treating this small area as a separate object within the larger source. The islands were corrected in the segmentation image by an automated process. We masked each individual pixel that was surrounded by $N$ already masked pixels, and we repeated this process $M$ times. By experimentation, we found that $N=6$ and $M=20$ filled in the majority of the island-like structures with minimal changes to any other region. Finally, the images multiplied by the mask were visually inspected, and any regions that needed any further masking were masked using IMEDIT. These regions were mostly large-scale islands of unmasked pixels that were not removed by our automated procedure. Less than $2 \%$ and $0.5 \%$ of the pixels in Abell 1413 and MKW 7 respectively were removed manually. The fraction of the images that was masked at this point is $43.4 \%$ for Abell 1413, and 52.36\% for MKW 7. Figure 9 shows a sub-region of the MKW 7 image that contains stars and galaxies through each step of the masking process.

\subsection{Final Sky Subtraction, Masking and Large-scale Flat-Fielding Errors}

Accurate sky subtraction is crucial to determine the true amount of intracluster starlight in each cluster, and is one of the dominant sources of error in our analysis. We now find a more accurate sky level for each cluster by using the masked image. We first bin up the entire image into squares of $49 \times 49$ pixels. For each bin, we calculate a robust average (Morrison, Boroson, \& Harding 1994), ignoring all masked pixels; the results are displayed in Figures 10 and 11, respectively. It is important to note that the entire greyscale range displayed in Figures 10 and 11 is \pm 5 ADU from the sky level, which corresponds to a surface brightness of $\mu_{\mathrm{V}}=26.7 \mathrm{mag} \operatorname{arsec}^{-2}$, or 5.5 magnitudes below the sky level.

Several distinct features are apparent in these binned images. First, the bright central portion of the $\mathrm{cD}$ is completely masked. Then, as the distance from the $\mathrm{cD}$ increases, there is an annulus of bins where almost all pixels are masked, except for a few pixels that are significantly below the median value of the total number of pixels in the bin. This is the origin 
of the lower-flux "ring" seen around each cD galaxy and is simply an artifact of masking the $\mathrm{cD}$. As we move even further outwards, we find a region where the $\mathrm{cD}$ halo is still detectable, but it has dropped below the surface brightness at which SExtractor masks individual pixels $\left(\mu_{\mathrm{V}} \sim 27.3 \mathrm{mag} \operatorname{arsec}^{-2}\right)$. Finally, at the edges, the flux comes to a more or less constant value.

However, in the case of Abell 1413, there is an additional low-surface brightness feature stretching along the right side of the frame at an amplitude of $\sim 1$ ADU $\left(\sim \mu_{\mathrm{V}}=28.5\right.$ mag $\left.\operatorname{arsec}^{-2}\right)$. This feature is almost certainly instrumental in nature, corresponding to the vignetting of the southern region of the T2KA chip by the $2.1 \mathrm{~m}$ guide camera (Massey et al. $2000)^{5}$. This flat-fielding residual is small, but since it is systematic in nature, we chose to mask all pixels in the Abell 1413 image with $\mathrm{x}>$ 1300. For MKW 7, we see no evidence for this effect, and so we do not mask further in this case.

To better determine our sky-values, and to measure our large-scale flat-fielding errors, we fit and subtract a plane from each masked, binned cluster image, using the IMSURFIT task in IRAF. We took care to use regions on each image that are well away from the central $\mathrm{cD}$. The mean corrections from this step are small: less than 0.5 ADU for both clusters on average. However, we emphasize that this process will remove any ICL that covers the entire image. We then created a histogram of sky values in $49 \times 49$ pixel bins well away from the center of each cluster. We also required that the bins contain at least 200 unmasked pixels to be included in the histogram. There are 653 such bins in Abell 1413 and 746 bins in MKW 7. These histograms are displayed in Figure 12. The width of the histograms provides a measure of our uncertainties due to large-scale flat-fielding errors, and the faint outer wings of stars and galaxies that remain unmasked, even after the involved procedure above. We find that the large-scale flat-fielding error for both image is conservatively $1 \mathrm{ADU}^{6}$, which corresponds to an uncertainty of $0.11 \%$.

\subsection{Constructing the surface brightness profile}

We now un-mask the region around the central cD galaxy, and re-build the mask leaving the $\mathrm{cD}+$ intracluster light intact. We proceed as follows: we first use the ELLIPSE task in IRAF/STSDAS (Busko 1996), based on the algorithms of Jedrzejewski (1987), to obtain an approximate geometrical model of $\mathrm{cD}+$ intracluster light. We then subtract this model

\footnotetext{
${ }^{5}$ this document is available at http://www.noao.edu/kpno/kpno.html

${ }^{6}$ equivalent to $1.3 \sigma$ if the distribution is Gaussian
} 
from the data, and mask all the stars and galaxies that are superimposed over the $\mathrm{cD}$ using the same techniques as before. With this improved mask, we create a better model using ELLIPSE, and repeat the process until the residuals from the subtracted image are minimized. This process was repeated seven times for each cluster.

In the case of MKW 7, a complication occurs at this step. There is a bright $\left(m_{v} \approx 11.9\right)$ saturated star that lies within $21^{\prime \prime}$. 1 of the nucleus. Naturally, we mask the inner regions of this star, but because it is so bright, its radius of influence extends over much of MKW 7's nucleus. To remove its influence on our surface photometry, we found its magnitude from a series of 10 second linearized exposures taken at the same time as our surface brightness data. We then subtracted the magnitude scaled PSF ( $\$ 6.1)$ from the MKW 7 data. This subtraction is good to 0.05 magnitudes, and that error is incorporated into the error model for those bins. Because the bright core of the star is masked, the increase in the error is actually quite small.

We now bin up the un-masked data into regions whose size varies from a resolution element $(5 \times 5$ pixels $)$ near the cluster center, to the maximum $49 \times 49$ pixel bin at the edges, using the robust mean as before. The binsize was increased exponentially with distance in the $\mathrm{x}$ and $\mathrm{y}$ directions, so that the signal-to-noise ratio did not strongly vary from the inner to outer regions. The scale length was 100 pixels in each direction. There are 11,037 such bins in the Abell 1413 image, and 7,925 bins in the MKW 7 image. We next transform the mask-weighted $\mathrm{x}$ and $\mathrm{y}$ coordinates of each bin to the appropriate elliptical coordinates. We do this by taking the best results from the ELLIPSE runs above, which consist of a table of the best-fitting elliptical isophotes as a function of semi-major axis (for full details, see Jedrzejewski 1987) For each bin, we adopt the ellipticity and position angle from the nearest elliptical isophote from the ELLIPSE table. In some cases, the ellipticity and position angles shifted abruptly in a non-physical manner. Therefore, we boxcar smoothed the ellipticity and position angle tables before applying them to our data. With the $\mathrm{x}$ and $\mathrm{y}$ coordinates from our binning program, and the adopted, boxcar smoothed ellipticity and position angles from the ELLIPSE runs, we have now defined a unique ellipse for each bin, with a semi-major axis $a$ and semi-minor axis $b$, with our bin at an eccentric angle $E$. The surface brightness profiles for Abell 1413, and MKW 7 are displayed in Figure 13. In all cases, we define our radial coordinate $r$ as the geometric mean of the semi-major and semi-minor axes: $r=\sqrt{a b}$.

\subsection{Limits to Our Precision}

The flux error model is described in detail in the Appendix. To illustrate, we work through the errors in a $5 \times 5$ pixel bin, located 98 pixels in radius from the center of Abell 1413 . 
In this bin, the mean number of counts is 40.9 \pm 1.9 ADU above the sky level. The errors are summarized in Table 1. At large radii from the $\mathrm{cD}$, the largest sources of error are large-scale flat-fielding errors, which are systematic, and do not depend on bin size. Our errors at these large radii are $\sim 1.2 \mathrm{ADU}$ per bin. Therefore, for a surface brightness bin to have a signal-to-noise ratio of at least five, it must have a mean value of at least 6 ADU,

which corresponds to a surface brightness of $\mu_{\mathrm{V}}=26.5 \mathrm{mag} \operatorname{arsec}^{-2}$. The signal-to-noise ratio approaches unity at $\mu_{\mathrm{V}}=28.3 \mathrm{mag} \operatorname{arsec}^{-2}$.

There is an additional source of error due to the coordinate transformation via the ELLIPSE fits. Any error in the ellipticity or position angle adopted will translate to an error in ellipsoidal radius. To quantify this error, we propagated the error bars for the ellipticity and position angle derived from the ELLIPSE task through our transformation formulae. The error is typically $1.0 \%$ in the radial direction. Although this error seems small, it does have a significant impact on the errors for each bin. If we transform the error in the radial coordinates to the corresponding error in magnitudes, we find the error is typically 0.04 magnitudes, assuming that the light follows a $r^{1 / 4}$ law. To test the accuracy of the error bars from ELLIPSE, we simulated a series of images using tasks in the ELLIPSE package, and the parameters of MKW 7's best-fitting model. We then applied our error model to make noisy images from this model, re-ran ELLIPSE, and measured the dispersion in the measured parameters. We found that the ELLIPSE task gave reasonable error estimates, actually overestimating the error by about a factor of two. To be conservative, we adopt the ELLIPSE errors as they stand.

In short, we are confident that we have identified the major sources of error due to instrumentational, observational, and computational sources. A detailed, quantitative understanding of our errors is crucial for accurate measurement of the surface brightness profile. One distinct advantage of this rigorous approach is that we can search for non radial features in our data that a simple average would miss.

\subsection{Comparison with Published Results}

In order to directly compare our data against previously published results, we first azimuthally average our surface brightness bins. We then compare our Abell 1413 data to the $V$ photographic surface photometry of Schombert (1986) and our MKW 7 data to the $B$ photographic data of Morbey \& Morris (1983). The results are plotted in Figures 14 and 15 , respectively.

For MKW 7, after a displacement of 1.1 magnitudes to account for passband differences 
between the two images, and the average color of the galaxy light, the difference between the two data sets is less than 0.1 mags everywhere but at very large radii. The Morbey \& Morris (1983) data is slightly brighter than our data at radii between 16 and 23 arcseconds; this may be due to the influence of the bright star 21". 1 away from MKW 7's nucleus. Otherwise, the agreement is very good.

For Abell 1413 however, our data do not agree as well with that of Schombert (1986). There are clear systematic differences between the two radial profiles at both large and small radii. In the inner portions, the Schombert (1986) data are systematically brighter by up to a magnitude. This may be due to difficulties in transforming measured photographic densities to magnitudes at higher flux levels. As evidence of this, we compare our CCD data at small radii to the CCD data of Schneider, Gunn, \& Hoessel (1983); which consists of a Gunn $r$ radial surface brightness profile of Abell 1413, taken under similar seeing conditions ( $1^{\prime \prime}$. 22 versus $1^{\prime \prime}$. 47). After a displacement of 0.3 magnitudes to account for the cD's color (plotted in Figure 16), the two CCD data sets are in excellent agreement.

At large radii, our measurements find systematically less flux than the Schombert (1986) data. Figure 17 shows this region of discrepancy in more detail. Unfortunately, the area of comparison is exactly the region where our signal-to-noise is rapidly decreasing and where sky-subtraction dominates the errors. Figure 17 also shows the effect on our measured surface brightness profile if we had over-estimated the sky value by $1 \mathrm{ADU}$ (if, for example, our field of view had not reached the sky). As can be clearly seen, even a small error in our sky can alter the results dramatically in this case. Therefore, although we measure less flux than Schombert (1986) in this region, we cannot convincingly argue that our data is favored. Due to the large angular coverage of the photographic data, the sky subtraction of Schombert (1986) may be more complete than our own.

Could the discrepancy between our data and those of Schombert etal be due to the presence of color gradients in the $\mathrm{cD}$ envelope? Since we have not included color terms in our transformation from observed Washington $\mathrm{M}$ to Johnson $\mathrm{V}$, any underlying color gradient could systematically affect our photometry and produce the observed discrepancy between the two datasets. In practice, however, the effect is small. Mackie, Visvanathan, \& Carter(1990) have made a study of color gradients in central dominant galaxies, and they have found that the gradients are small, generally less than 0.2 magnitudes in $(\mathrm{B}-\mathrm{V})$ over the entire radial range observed. Additionally, Mackie (1992) studied the colors of cD envelopes and found that their color profiles were also quite flat. Since we have calibrated our data for the mean $(\mathrm{B}-\mathrm{V})$ color of $\mathrm{cD}$ galaxies $(\mathrm{B}-\mathrm{V}=1.0)$, and given color term derived in $\S 5.4$, this means that at most, 0.04 magnitudes of the offset can be attributed to color terms in our CCD data. Therefore, color terms cannot solely account for the discrepancy. 


\subsection{The Surface Brightness Profiles of Abell 1413 and MKW 7}

We now fit the surface brightness profiles of Abell 1413 and MKW 7 using the Sersic (1968) profile:

$$
I(r)=I_{e} 10^{-b_{n}\left[\left(r / r_{e}\right)^{1 / n}-1 .\right]}
$$

where $b_{n}$ is a constant chosen so that half the total luminosity predicted by the law is interior to $r_{e}$, and is well approximated by the relation $b_{n}=0.868 n-0.142 . I_{e}$ is the intensity at the effective radius. Because $\mathrm{cD}$ halos may exist as excesses above a best-fitting Sersic profile, we first fit the inner regions of the surface brightness profiles $\left(\mu_{\mathrm{V}}<26\right.$. $)$. Additionally, to ensure that our results are not affected by seeing, we ignore all data that has a radius less than three times the measured FWHM of each image. We find that the best-fitting $n$ values for both Abell 1413 and MKW 7 are indistinguishable from $n=4$, the de Vaucouleurs profile (de Vaucouleurs 1948). The best-fitting $r^{1 / 4}$ parameters for the inner regions of Abell 1413 and MKW 7 are given in Table 2. We note that the reduced $\chi^{2}$ values for both fits are quite high: 3.2 for Abell 1413 and 15.9 for MKW 7. We discuss the causes of this in the next section.

\subsection{Deviations from the $r^{1 / 4}$ law:}

There are two possible explanations for why the fits have high reduced $\chi^{2}$ values: 1) we have underestimated our error bars substantially or 2) there are real deviations in each $\mathrm{cD}$ galaxy from an $r^{1 / 4}$ law. As we have stated earlier, we believe we have addressed all significant sources of error, including sky subtraction and other systematic sources. Therefore, we turn to possible deviations as the source of the large residuals.

On large angular scales, it is believed that excesses above the $r^{1 / 4}$ law for $\mathrm{cD}$ galaxies at large radii are due to what was classically called cD envelopes (Tonry 1987; Schombert 1992). We now search for the presence of such envelopes in our data. After subtracting the best-fit $r^{1 / 4}$ law found above for each cluster, we fit another simple model to our data. We assume that at a semi-major axis smaller than a radial scale $r_{\text {cutoff }}$, that there is no measurable excess in the surface brightness profile over the $r^{1 / 4}$ law. At radii larger than

$r_{\text {cutoff }}$, there is an excess above the best-fitting de Vaucouleurs model that is linear with $r^{1 / 4}$, and has a slope $\beta$ :

$$
\mu=0 ;\left(r \leq r_{\text {cutoff }}\right)
$$




$$
\mu=\beta\left[r^{1 / 4}-r_{\text {cutoff }}^{1 / 4}\right] ; \quad\left(r>r_{\text {cutoff }}\right)
$$

We emphasize that this parameterization is not intended to act as a physical model, but rather a simple way of quantifying any luminosity excess. We now fit our data using standard least-squares methods to this model. To obtain a robust result, we limit our fitting to where our data has a signal-to-noise of five or greater $\left(\mu_{\mathrm{V}}<26.5 \mathrm{mag} \operatorname{arsec}^{-2}\right)$.

The results are plotted in Figure 18, and the best-fitting parameters are given in Table 3, columns 1-5. We find that both Abell 1413 and MKW 7 have clear excesses above the $r^{1 / 4}$ law. This is not unexpected for Abell 1413 (Schombert 1986), but the excess for MKW 7 is completely unexpected. Thuan \& Romanishin (1981) studied the surface brightness profile of nine brightest cluster members in MKW/AWM clusters and found that all of them followed an $r^{1 / 4}$ profile out to large radii. Because of the lack of excess, Thuan \& Romanishin (1981) then argued that such brightest cluster galaxies were not "true" cD galaxies. In their interpretation, $\mathrm{cD}$ envelopes are formed from galaxy collisions, and hence will only be found in rich clusters.

Here, we have found the exact opposite behavior: the poor cluster has a definite excess. In the case of Abell 1413, we also find a clear excess, but it is significantly smaller than that found by Schombert (1986). The lower inferred excess is due to the discrepancies at both large and small radius between our data and those of Schombert (1986). The steeper inner profile of Schombert (1986) results in a steep $r^{1 / 4}$ fit, enhancing the excess at large radius. Our inner data points produce a shallower $r^{1 / 4}$ fit, and our outer data show lower surface brightnesses than Schombert (1986). Both effects significantly reduce the inferred luminosity excess over the single $r^{1 / 4}$ fit.

To quantify these effects, we integrated our best-fitting $r^{1 / 4}$ profiles, and our model for the excesses. The results are given in Table 3, columns 6-8. We find that the fraction of total luminosity in the excess component at the radius where our data reaches a signal-to-noise of five is $13 \%$ for Abell 1413, and 21\% for MKW 7. We then extrapolated our value for the Abell 1413 excess out to very large radii, in order to compare with the results of Schombert (1988). We stress that this extrapolation is very uncertain, as the value strongly depends on the accuracy of the slope $\beta$, and whether such a simple model is reasonable at very large radii. We find that the fraction of excess luminosity to the total luminosity increases to $45 \%$. However, we also find that the total derived luminosity is a factor of $\sim 2$ less, and the luminosity of the excess component is a factor of $\sim 3$ less than Schombert (1988) found.

With the addition of the envelopes to our models of the surface brightness distribution in Abell 1413 and MKW 7, the reduced $\chi^{2}$ values show a large improvement: 2.1 for Abell 1413 
and 4.3 for MKW 7. However, given that we have high confidence in our error models, such high reduced $\chi^{2}$ values are still unacceptable. Therefore, there are still additional deviations from the elliptically symmetric flux model we have adopted.

This result is supported by independent numerical results from the ELLIPSE program fits. Numerous surface brightness studies of elliptical galaxies have shown that the surface brightness profiles do not follow perfect ellipses (e.g., Jedrzejewski 1987; Peletier et al. 1990). These non-elliptical terms are often parameterized as the third and fourth-order terms of a Fourier series:

$$
I(\theta)=I_{0}\left(\sum_{n=3}^{4} A_{n} \sin (n \theta)+\sum_{n=3}^{4} B_{n} \cos (n \theta)\right)
$$

where $I_{0}$ is the mean intensity of the elliptical isophote, and $\theta$ is the angle around the ellipse. The ELLIPSE program calculates these parameters automatically, and in Figure 19, we plot the derived $\mathrm{A}_{3}, \mathrm{~B}_{3}, \mathrm{~A}_{4}$ and $\mathrm{B}_{4}$ terms for Abell 1413 and MKW 7. The terms are expressed as the Fourier amplitudes normalized by the semi-major axis and the isophotal gradient. The third order terms describe asymmetries in the light profile, while the 4th order term in particular, A4 - describes "disky" (negative A4) or "boxy" (positive A4) isophotes. In both clusters, there are multiple regions where these terms are significantly non-zero, with amplitudes $(\sim 5 \%)$ much greater than those customarily seen in normal ellipticals $(0.5 \%$; Jedrzejewski 1987; Peletier et al. 1990).

In elliptical galaxies, these higher order isophotal coefficients are often used to search for the presence of disks or the effects of discrete mergers (e.g., Bender et al. 1989; Rix \& White 1990). In the distribution of ICL, which extends out to hundreds of kpc, this interpretation needs to be modified. To determine what causes these non-elliptical components in our data, we subtract the best-fitting elliptical isophotal profiles, including both the $r^{1 / 4}$ law and the excess component from both clusters, and then examine the residuals for bins that are brighter than $\mu_{\mathrm{V}}=26.5$ mag arsec $^{-2}$. These residuals are displayed in Figures 20 and 21.

In both cases, the elliptical fits break down near the center of the $\mathrm{cD}$ galaxy. This is not unexpected: the number of unmasked pixels to fit are very few, and the ELLIPSE algorithms are known to systematically underestimate the ellipticity of galaxies in the very center (Jedrzejewski 1987). In the case of MKW 7, the situation is particularly bad due to the bright star and galaxies very near the nucleus. However, even if we exclude these inner regions, and a handful of bins that have clearly discordant flux, the $\chi^{2}$ values for the fits are still deviant: 1.49 for Abell 1413 and 1.86 for MKW 7.

In the case of Abell 1413, there appears to be a series of positive and negative residuals, at a radial scale of $\sim 36^{\prime \prime}$. These residuals might be caused by a low surface brightness bridge between two large cluster galaxies present at that radius. Although there are tantalizing hints 
of diffuse tidal features in this residual image, none of them are clear enough to be definitive detections. However, for MKW 7, there is a plume-like feature clearly seen in the residual image. This feature has a mean surface brightness of $\mu_{\mathrm{V}}=25.6 \pm 0.2 \mathrm{mag} \mathrm{arsec}^{-2}$, and is approximately $61^{\prime \prime}$ long $(\sim 32 \mathrm{kpc})$. It is approximately $32^{\prime \prime}(\sim 17 \mathrm{kpc})$ in width at the base of the plume, narrowing near the tip to $\sim 24^{\prime \prime}(\sim 12 \mathrm{kpc})$. The presence of this plume drives the ELLIPSE fits to generate a $\cos (n \theta)$ residual all the way around the ellipse, and is the main cause of the high reduced $\chi^{2}$ found for MKW 7. If we approximate this plume as triangular in shape, we find a total magnitude of $\mathrm{V} \sim 18$. Given our adopted distance modulus to MKW 7, this is equal to the luminosity of a small galaxy $\left(M_{\mathrm{V}} \sim-17\right)$. This plume is clear evidence for ongoing tidal activity in MKW 7, and is similar in appearance to tidal debris found in the halo of M 87 (Weil, Bland-Hawthorn, \& Malin 1997).

\subsection{Geometric properties of Abell 1413 and MKW 7}

From our adjusted fits from the ELLIPSE program, we also obtain the geometric parameters of the $\mathrm{cD}+$ intracluster light, such as the ellipticity and the position angle of the best-fitting ellipses. Those results are plotted in Figure 22. For Abell 1413, we have compared our results with those of Porter, Schneider, \& Hoessel (1991, hereafter PSH), and find good agreement over the range of radii we have in common.

The ellipticity of both clusters increases steadily with radius. This is in good agreement with the results of PSH, who found an identical trend at smaller radii with a sample of 175 brightest cluster ellipticals. However, the ellipticity $(\approx 0.8)$ of Abell 1413 at large radii is extraordinary. At a radii of $64 \mathrm{kpc}$ (assuming $\mathrm{H}_{0}=60 \mathrm{~km} \mathrm{~s}^{-1} \mathrm{Mpc}^{-1}, \mathrm{q}_{0}=0.5$ ), PSH found the average ellipticity of brightest cluster ellipticals to be $\approx 0.4$, and the maximum ellipticity to be 0.59, making Abell 1413 the most flattened brightest cluster galaxy ever measured. However, the maximum value of PSH's ellipticity distribution is the value for Abell 1413. It is therefore unclear whether the large ellipticity of Abell 1413 is peculiar to this cluster, or whether ellipticity for many brightest cluster galaxies continues to increase beyond the radial limits observed by $\mathrm{PSH}$. In contrast, the ellipticity distribution of MKW 7 is much more typical of that previously observed: a smooth rise to a maximum value of $\approx 0.4$. This might be due to the fact that we probe a smaller range of physical radii in MKW 7, compared to Abell 1413.

In terms of position angle, Abell 1413 has only small isophote twists: less than two degrees change overall. MKW 7 has a large, but not extraordinary twist of twenty degrees near the center, followed by a gradual change in position angle out to large radius. These patterns are common for brightest cluster ellipticals (Porter, Schneider, \& Hoessel 1991). 


\section{The Search For Tidal arcs}

With the large scale properties of intracluster light $+\mathrm{cD}$ galaxy established, we now focus on searching for smaller scale tidal debris arcs. For the purposes of this search, we define an arc as an extremely elongated (ellipticity $\geq 0.5$ ) discrete object that can be detected visually.

We take the residual images constructed in $\S 6.7$ and visually search them for the presence of any tidal debris arc structures. We took two steps to ensure that residuals from the $\mathrm{cD}$ subtraction process were not mistakenly identified as tidal arcs. First, we avoided the very inner $10^{\prime \prime}$ radius of the center of each cluster, where the residuals are the strongest. Second, we also demanded that the arc candidate be visible in the unsubtracted cluster image, as well as the cD-subtracted image. We found a total of five arc-like candidates in Abell 1413, and one candidate in MKW 7. These arc-candidates are shown in Figures 23 and 24, respectively.

The candidate arcs were then analyzed using the SExtractor software package. Astrometry for the central portion of the arcs was derived using the USNO-A 2.0 astrometric catalog (Monet et al. 1996; Monet 1998), and the FINDER astrometric package from IRAF, and are accurate to $0.3^{\prime \prime}$. The results of the analysis are given in Table 4 . We compare these results to the tidal arcs previously found in the Coma and Centaurus clusters (Trentham \& Mobasher 1998; Gregg \& West 1998; Calcańeo-Roldán, et al. 2000), whose properties are presented in Table 5. Since these other observations are taken in different filters than $\mathrm{V}$, a color correction must be applied. Gregg \& West (1998) found optical colors of $\mathrm{B}-\mathrm{V} \approx 0.9$, $\mathrm{V}-\mathrm{R} \approx 0.6, \mathrm{~V}-\mathrm{I} \approx 1.2$ for their debris arc candidate, typical of old stellar populations, so we adopt these colors for comparison purposes.

We find that the arc candidates found in Abell 1413 and MKW 7 are significantly shorter ( 10-20 kpc compared to $100 \mathrm{kpc}$ ), and generally have higher surface brightness $\left(\mu_{\mathrm{V}} \sim 25.5 \mathrm{mag} \operatorname{arcsec}^{-2}\right.$ compared to $26 \mathrm{mag} \operatorname{arsec}^{-2}$ ) than the tidal debris arcs seen in Coma and Centaurus. We conclude that, down to a limiting surface brightness of $\mu_{\mathrm{V}}=26.5$ mag $\operatorname{arsec}^{-2}$, there are no tidal debris arcs longer than $30 \mathrm{kpc}$ in either Abell 1413 or MKW 7. Given the depth of our images, if either cluster contained long arcs such as those detected in Coma and Centaurus, we would have detected them. The fact that we do not detect them, particularly in a rich cluster like Abell 1413, argues for real differences in the intrinsic ICL properties of massive clusters.

So, what are these smaller arc-like objects that we do detect? Arc candidates 1-3 of Abell 1413 lie tangentially to the $\mathrm{cD}$ galaxy, implying that these arcs may be due to strong gravitational lensing. Gravitational arcs in clusters at this redshifts are uncommon (Fort \& Mellier 1994), but some have been observed (e.g., Campusano, Kneib, \& Hardy 
1998; Blakeslee \& Metzger 1999), and theoretical calculations indicate that they should be detectable (Natarajan \& Kneib 1997; Cypriano et al. 2001). Spectroscopic follow-up observations will be needed to prove whether these arcs are gravitational in nature. Arc candidates 4-5 of Abell 1413 may be other gravitational arcs, or genuine tidal debris. For the MKW 7 arc candidate, due to the lower redshift of the cluster, and the fact that the MKW 7 arc candidate is extended in both dimensions, it is unlikely that this arc is due to gravitational lensing. We may be witnessing the early stages of the disruption of a dwarf cluster galaxy, but without a redshift to assure cluster membership, this conclusion is perhaps premature. While the MKW 7 arc candidate lies within, and is perpendicular to the tidal plume detected there, this is likely to be coincidental.

\section{Discussion and Summary}

We have performed deep surface photometry of two galaxy clusters of greatly varying richness: Abell 1413 and MKW 7. We find that both galaxy clusters contain intracluster light out to large radii. The $\mathrm{cD}$ envelopes of both clusters follow an $r^{1 / 4}$ profile over a large range in radius, but also show an excess of diffuse light at the largest radii. We also find evidence for substructure in the ICL in MKW 7 in the form of a tidal plume and a single small arc structure, and a set of small arc-like structures in Abell 1413 which may either be tidal in origin, or possibly due to gravitational lensing.

The accepted view of cD galaxies (Tonry 1987; Schombert 1992) is that brightest cluster galaxies in rich clusters have large excesses in their surface brightness profiles over an $r^{1 / 4}$ law, and are denoted as type $\mathrm{cD}$, while brightest cluster galaxies in poor clusters do not have an excess, and are usually given a different designation (type D). However, this view was established with photographic data, and newer CCD observations may cause this view to be revised. Multiple authors using CCDs have observed brightest cluster galaxies in rich clusters and have found that they follow an $r^{1 / 4}$ law out to very large radii (Uson, Boughn, \& Kuhn 1991; Scheick \& Kuhn 1994; Gonzalez et al. 2000). In our particular case, we observed a classical "cD envelope" cluster (Abell 1413), and detected a much smaller envelope than the original photographic data found. In addition, we found a clear excess in a poor cluster (MKW 7), where photographic data of similar clusters has found no excess (Thuan \& Romanishin 1981). Although more observations are needed, especially with clusters observed both with photographic data and CCDs, it seems clear that the exact nature of $\mathrm{cD}$ envelopes needs to be re-evaluated.

If some rich clusters have ICL that follow $r^{1 / 4}$ profiles, while others do not, it is possible that we can use the profiles to place constraints on ICL formation mechanisms. As mentioned 
in $\S 1$, while violent relaxation is known to produce a $r^{1 / 4}$ profile, tidal stripping may produce a wider variety of profiles, depending on the distribution of energy and angular momentum of the stripped population. This would imply that a cluster whose ICL followed an $r^{1 / 4}$ profile is dynamically relaxed, and produced the majority of its ICL in the process of cluster formation, when the gravitational potential is rapidly changing. In contrast, deviations from the $r^{1 / 4}$ law would imply that intracluster star production is ongoing, and the cluster is not dynamically relaxed. The fact that our two clusters, which are quite disparate in mass, both show good $r^{1 / 4}$ profiles to large radius favors models where the bulk of ICL is produced early during cluster collapse. However, ongoing stripping does occur, as evidenced by the tidal plume and small luminosity excesses in the very outer regions of the clusters.

Separate from the radial profiles of ICL is the presence or absence of tidal debris (plumes and arcs). In our observations of MKW 7, we have clearly found evidence for a tidal plume, much like that seen in M 87 by Weil, Bland-Hawthorn, \& Malin (1997). The luminosity is small, like that of a small spiral galaxy, but reinforces the finding that even for poor clusters, tidal stripping can be an important effect.

As for tidal debris arcs, we detected a number of possible short tidal structures, but no long tidal arcs such as those seen in Coma and Centaurus (Trentham \& Mobasher 1998; Gregg \& West 1998; Calcańeo-Roldán, et al. 2000). With a sample of only two galaxy clusters, it is premature to make any serious conclusions about the true frequency of long tidal debris arcs. It might be that smaller-scale tidal structure in galaxy clusters, such as that seen by Conselice \& Gallagher (1999) are generally more common in galaxy clusters than long tidal debris arcs. Long tidal arcs are dynamically delicate, and may be destroyed by the passage of another galaxy in the cluster. On the other hand, the lack of long tidal arcs might be due to the properties of the clusters studied. Abell 1413 appears to be dynamically evolved, and perhaps tidal debris arcs are less common in such systems. MKW 7 is a much poorer cluster, so encounters are less common. A larger sample of clusters is clearly needed for further progress.

We do note that the vast majority of tidal debris seen in cluster simulations (Moore et al. 1996; Dubinski 1998; Dubinski, Murali, \& Ouyed 2000) has a surface brightness much lower than our $\mu_{\mathrm{V}}=26.5 \mathrm{mag} \operatorname{arsec}^{-2}$ limit. The structures that we have observed so far may only be the brightest features in each cluster. Planned observations to deeper surface brightness limits will be important in the future.

We thank P. Durrell for giving us the inverse image idea and we especially thank K. Stanek and B. Mochesjka for their help with the T2KA linearity problem. We thank the KPNO staff, especially R. Probst, for their help with the scattered light patterns. We thank 
W. Pence for some early help, and his easy-to use CFITSIO routines, and we thank I. Busko and R. Jedrzejewski for their help with the ELLIPSE program. We also thank an anonymous referee for useful comments that improved the presentation of this paper.

This work is supported by NSF through grants AST-9876143 (JCM) and AST-9624542 (HLM) and by NASA through grant NAG5-7019 (JCM). Funding was also given by the Research Corporation's Cottrell Scholarships (JCM and HLM). 


\section{A. The Error Model}

It is necessary to have accurate error estimates of our surface photometry in order to perform the model fitting. Unlike earlier photographic work, deep CCD surface photometry allows us to quantify measurement errors. Measurement errors arise from CCD behaviors such as readout noise and flat-fielding, as well from sky noise. Each error contribution will be addressed below.

\section{B. Readout Noise}

The readout noise per exposure is 1.1 ADU. By combining 9 images for Abell 1413, and 12 images for MKW 7 with a median, we are able to reduce the effective read noise to

$$
R_{e f f}=1.1 A D U \frac{1.22}{\sqrt{N_{G}}}
$$

The factor of 1.22 is due to the lower efficiency of a median over a mean (see Morrison, Boroson, \& Harding 1994, Section 3.2.1).

\section{Photon Noise}

For $C$ ADU in a given pixel, the photon noise is $(\mathrm{C} / g)^{1 / 2}$, where $g$ is the gain. Combining 9 and 12 images respectively using a median reduces the photon noise to

$$
\sigma_{\text {Poisson }}=\frac{1.22}{\sqrt{N}} \frac{\sqrt{C}}{\sqrt{g}}
$$

\section{Linearity Errors}

As mentioned in $§ 5.2$ above, the T2KA chip has a known non-linearity. We have corrected for this effect, but the parameters used for the correction do not have infinite precision, and so our correction has errors. The error in flux can be derived as follows:

$$
\sigma_{\text {linearity }}^{2}=\sigma_{c 1}^{2} C_{s k y}^{2}+\sigma_{c 2}^{2} \frac{C_{s k y}^{4}}{(32767)^{2}}+\sigma_{c 3}^{2} \frac{C_{s k y}^{6}}{(32767)^{4}}
$$

where $C_{s k y}$ is the sky-subtracted flux. Since this correction is small, we apply it only to the flux, and not to any other calibration image. 


\section{E. Flat-Fielding Errors}

In principle, the only limit to the precision of the combined flat-field images is the photon noise in the individual flat-field images. This small-scale variation is

$$
\sigma_{s f f}=\frac{\sqrt{C_{s}}}{\sqrt{g}} \frac{1.22}{\sqrt{N_{f}}} \frac{1.22}{\sqrt{N_{g}}}
$$

where $C_{s}$ is the number of counts in the final, combined master sky flat image, $\mathrm{g}$ is the gain, $N_{f}$ is the number of individual sky flats used to make the master sky flat, and $\mathrm{N}_{g}$ is the number of individual galaxy images used to make the final galaxy image. The sky counts $C_{s}=941 \mathrm{ADU}$, the gain is $3.6 \mathrm{ADU}$, the number of sky images is 20, and the number of galaxy images is 9 and 12, respectively. The percentage errors are $0.191 \%$ for Abell 1413 , and $0.166 \%$ for MKW 7 .

In practice, the small-scale flat-fielding errors are not the only flat-fielding error we have. There are also large-scale variations which arise from the variation of the sky brightness across the image, instrumental effects such as flexure, and from the wings of bright stars and galaxies that were not completely removed by combining the individual sky flats. Normally, to measure this effect, we prefer to divide our sky flats into two sub-samples, create two sky flat images from those sub-samples, and then find the standard deviation of the ratio of the two created flats. However, we only have twenty sky images, and dividing them up into two ten image sub-samples would be too noisy for a realistic measurement.

Instead, we masked each image, and constructed a histogram of sky values $(\S 6.2)$. With $49 \times 49$ pixel bins, the noise between each bin is completely dominated by large-scale flatfielding errors, and the faint wings of unmasked objects. We found an error of 1.0 ADU, which corresponds to a fractional error of $1 / 941.0$, or a percentage error of $0.11 \%$.

\section{F. Surface Brightness Fluctuations}

For ultra-deep surface brightness observations of nearby galaxies, a major source of error arises from intrinsic surface brightness variations (Tonry \& Schneider 1988). For our distant galaxy clusters (see eq 10 of Tonry \& Schneider (1988)), such an effect is completely

negligible compared to our other errors. 


\section{REFERENCES}

Abell, G. O., Corwin, H. G., \& Olowin, R. P. 1989, ApJS, 70, 1

Albert, C. E., White, R. A., \& Morgan, W. W. 1977, ApJ, 211, 309

Arnaboldi, M., Freeman, K. C., Méndez, R. H., Capaccioli, M., Ciardullo, R., Ford, H., Gerhard, O., Hui, X., Jacoby, G. H., Kudritzki, R. P., \& Quinn, P. J., 1996, ApJ, 472,145

Bahcall, N. A. 1980, ApJ, 238, 117

Beers, T. C., Kriessler, J. R., Bird, C. M. \& Huchra, J. P. 1995, AJ, 109, 874

Bender, R., Surma, P., Doebereiner, S., Moellenhoff, C., \& Madejsky, R. 1989, A\&A, 217, 35

Bernstein, G. M., Nichol, R. C., Tyson, J. A., Ulmer, M. P., \& Wittman, D. 1995, AJ, 110, 1507

Bertin, E. \& Arnouts, S. 1996, A\&AS, 117, 393

Blakeslee, J.P. \& Metzger, M.R. 1999, ApJ, 513, 592

Busko, I. C. 1996, ASP Conf. Ser. 101: Astronomical Data Analysis Software and Systems $\mathrm{V}, 5,139$

Calcáneo-Roldán, C. , Moore, B. , Bland-Hawthorn, J. , Malin, D., \& Sadler, E. M. 2000, MNRAS, 314, 324

Campusano, L. E., Kneib, J.-P. \& Hardy, E. 1998, ApJ, 496, L79

Ciardullo, R., Jacoby, G. H., Feldmeier, J. J., \& Bartlett, R. E. 1998, ApJ, 492, 62

Ciardullo, R., Feldmeier, J. J., Krelove, K., Jacoby, G. H., \& Gronwall, C. 2002, ApJ, 566, 784.

Conselice, C. J. \& Gallagher, J. S. 1999, AJ, 117, 75

Cypriano, E. S. et al. 2001, AJ, 121, 10

de Vaucouleurs, G. 1948, Ann. Astrophys, 11, 247

Dressler, A. 1984, ARA\&A, 22, 185 
Dubinski, J. 1998, ApJ, 502, 141

Dubinski, J., Mihos, J. C., \& Hernquist, L. 1996, ApJ, 462, 576

Dubinski, J., Mihos, J. C., \& Hernquist, L. 1999, ApJ, 526, 607

Dubinski, J., Murali, C., \& Ouyed, R. 2000, unpublished preprint

Durrell, P., Ciardullo, R., Feldmeier, J. J., Jacoby, G. H., \& Sigurdsson, S. 2002, ApJ, in press

Feldmeier, J. J. 2000, Ph.D. Thesis, Penn State University

Feldmeier, J. J., Ciardullo, R., \& Jacoby, G. H. 1998, ApJ, 503, 109

Ferguson, H. C., Tanvir, N. R., \& von Hippel, T. 1998, Nature, 391, 461

Fort, B. \& Mellier, Y. 1994, A\&A Rev., 5, 239

Freeman, K. C. et al. 2000, ASP Conf. Ser. 197: Dynamics of Galaxies: from the Early Universe to the Present, 389.

Fry, A. M., Morrison, H. L., Harding, P., Boroson, T. A., 1999, AJ, 118, 1209

Gallagher, J. S., \& Ostriker, J. P. 1972, AJ, 77, 288

Gal-Yam, A. \& Maoz, D. 2000, Large Scale Structure in the X-ray Universe, Proceedings of the 20-22 September 1999 Workshop, Santorini, Greece, eds. Plionis, M. \& Georgantopoulos, I., Atlantisciences, Paris, France, p.359

Gonzalez, A. H., Zabludoff, A. I., Zaritsky, D., \& Dalcanton, J. J. 2000, ApJ, 536, 561

Grainge, K., Jones, M., Pooley, G., Saunders, R., Baker, J., Haynes, T., \& Edge, A. 1996, MNRAS, 278, L17

Gregg, M. D., \& West, M. J. 1998, Nature, 396, 549

Grundahl, F. \& Sørensen A. N. 1996, A\&AS, 116, 367

Jedrzejewski, R. I. 1987, MNRAS, 226, 747

Korchagin, V., Tsuchiya, T., \& Miyama, S. M. 2001, ApJ, 549, 244.

Krisciunas, K. 1997, PASP, 109, 1181 
Kudritzki, R.-P., Méndez, R. H., Feldmeier, J. J., Ciardullo, R., Jacoby, G. H., Freeman, K.C., Arnaboldi, M., Capaccioli, M., Gerhard, O., \& Ford, H.C. 2000, ApJ, 536, 19

Landolt, A. U. 1992, AJ, 104, 340.

Leir, A. A. \& van den Bergh, S. 1977, ApJS, 34, 381

Lynden-Bell, D. 1966, IAU Symp. 25: The Theory of Orbits in the Solar System and in Stellar Systems, 25, 78

Mackie, G. 1992, ApJ, 400, 65.

Mackie, G., Visvanathan, N., \& Carter, D. 1990, ApJS, 73, 637.

Massey, P. et al. 2000, New 2000 Direct Imaging Manual for Kitt Peak

Massey, P. \& Foltz, C. B. 2000, PASP, 112, 566.

Melnick, J., White, S. D. M., \& Hoessel, J. 1977, MNRAS, 180, 207

Mendez, R. H., Guerrero, M. A., Freeman, K. C., Arnaboldi, M., Kudritzki, R. P., Hopp, U., Capaccioli, M., \& Ford, H. 1997, ApJ, 491, L23

Merritt, D. 1983, ApJ, 264, 24

Merritt, D. 1984, ApJ, 276, 26

Miller, G. E. 1983, ApJ, 268, 495

Mochejska, B. J., Kaluzny, J., Stanek, K. Z., Sasselov, D. D., \& Szentgyorgyi, A. H. 2001, AJ, 121, 2032.

Monet, D. 1998, BAAS, 30, 1427

Monet, D., Bird, A., Canzian, B., Harris, H., Reid, N., Rhodes, A., Sell, S., Ables, H., Dahn, C., Guetter, H., Henden, A., Leggett, S., Levinson, H., Luginbuhl, C., Martini, J., Monet, A., Pier, J., Riepe, B., Stone, R., Vrba, F., \& Walker, R. 1996, USNO A - 1.0 a catalog of astrometric standards, U.S. Naval Observatory, Washington, D. C.

Morbey, C. \& Morris, S. 1983, ApJ, 274, 502

Morgan, W. W., Kayser, S., \& White, R. A. 1975, ApJ, 199, 545

Morgan, W. W. \& Lesh, J. 1965, ApJ, 142, 1364 
Morrison, H. L., Boroson, T. A. \& Harding P., 1994, AJ, 108, 1191

Morrison, H. L., Miller, E. D., Harding, P., Stinebring, D. R., \& Boroson, T. A. 1997, AJ, 113, 2061

Moore, B., Katz, N., Lake, G., Dressler, A., \& Oemler, A. 1996, Nature, 379, 613

Moore, B., Lake, G. \& Katz, N. 1998, ApJ, 495, 139

Natarajan, P. \& Kneib, J.-P. 1997, MNRAS, 287, 833

Oemler, A. 1976, ApJ, 209, 693

Peletier, R. F., Davies, R. L., Illingworth, G. D., Davis, L. E., \& Cawson, M. 1990, AJ, 100, 1091

Pence, W. 1999, in ASP Conf. Ser., Vol. 172, Astronomical Data Analysis Software and Systems VIII, ed. D. Mehringer, R. Plante, and D. Roberts (San Francisco: ASP), 487

Pilachowski, C. A., Africano, J. L., Goodrich, B. D., \& Binkert, W. S. 1989, PASP, 101, 707

Porter, A. C., Schneider, D. P., \& Hoessel, J. G. 1991, AJ, 101, 1561

Price, R., Duric, N., Burns, J. O., \& Newberry, M. V. 1991, AJ, 102, 14

Richstone D. O., \& Malumuth, E.M. 1983, ApJ, 268, 30

Rix, H. \& White, S. D. M. 1990, ApJ, 362, 52

Roach, F. E. \& Grodon, J. L. 1973, The Light of the Night Sky (Dordrecht, Reidel)

Scheick, X. \& Kuhn, J. R. 1994, ApJ, 423, 566

Schneider, D.P., Gunn, J.E. \& Hoessel, J.G. 1983, ApJ, 268, 476

Schombert, J.M. 1986, ApJS, 60, 603

Schombert, J.M. 1988, ApJ, 328, 475

Schombert, J. 1992, ASSL Vol. 178: Morphological and Physical Classification of Galaxies, 53

Sersic, J.-L. 1968, Atlas de Galaxias Australes (Cordoba: Obs. Astronomico)

Smith, H. A. 1981, AJ, 86, 998 
Stetson, P. B. 1987, PASP, 99, 191.

Struble, M. F. \& Rood, H. J. 1987, ApJS, 63, 555

Struble, M. F. \& Rood, H. J. 1999, ApJS, 125, 35

Theuns, T., \& Warren, S.J. 1997, MNRAS, 284, L11

Thuan, T. X., \& Romanishin 1981, ApJ, 248, 439

Tonry, J. L. 1987, IAU Symp. 127: Structure and Dynamics of Elliptical Galaxies, 127, 89

Tonry, J. \& Schneider, D. P. 1988, AJ, 96, 807

Trentham, N. \& Mobasher, B. 1998, MNRAS, 293, 53

Tyson, J. A., Kochanski, G. P. \& dell'Antonio, I. P. 1998, ApJ, 498, L107

Tyson, N. D., Bloser, P., Udalski, A., Syzmanski, A., Kaluzny, J., Kubiak, M., \& Mateo, M. 2002, ApJ, in preparation

Uson, J. M., Boughn, S. P., \& Kuhn, J. R. 1991, ApJ, 369, 46

van den Bergh, S. 1977, PASP, 89, 746

Vílchez-Gómez, R. 1999, ASP Conf. Ser. 170: The Low Surface Brightness Universe, 349

Víchez-Gomez, R., Pelló, R. \& Sanahuja, B. 1994, A\&A, 283, 37

Weil, M. L., Bland-Hawthorn, J., \& Malin, D. F. 1997, ApJ, 490, 664

Welch, G. A., \& Sastry G. N. 1971, ApJ, 169, L3

White, D. A. 2000, MNRAS, 312, 663

White, R. A., Bliton, M., Bhavsar, S. P., Bornmann, P., Burns, J. O., Ledlow, M. J., \& Loken, C. 1999, AJ, 118, 2014.

Whitmore, B. C., Forbes, D. A., \& Rubin, V. C. 1988, ApJ, 333, 542

Wild, W. J. 1997, PASP, 109, 1269

Zheng, Z. et al. (1999), AJ, 117, 2757

Zwicky, F. 1951, PASP, 63, 61 
Table 1. Errors in a $5 \times 5$ pixel bin with 40.9 ADU from Abell 1413 cD galaxy and 886 ADU from sky

\begin{tabular}{lll}
\hline \hline \multicolumn{1}{c}{ Source } & Error in ADU & \% Error \\
\hline Readout noise & 0.12 & 0.3 \\
Poisson Statistics & 1.5 & 3.7 \\
Linearity Error & 0.21 & 0.5 \\
Small-scale flat-fielding & 0.46 & 1.1 \\
Large-scale flat-fielding + Sky subtraction & 1.0 & 2.4 \\
\hline Total Error & 1.9 & 4.6 \\
\hline
\end{tabular}

Table 2. Best-fitting de Vaucouleurs parameters

\begin{tabular}{lllccc}
\hline \hline Cluster & $\begin{array}{c}\text { intercept of } \\
\text { best-fit (mag) }\end{array}$ & $\begin{array}{c}\text { slope of } \\
\text { best-fit }\left(\mathrm{mag} / \mathrm{r}_{\text {arcsec }}^{1 / 4}\right)\end{array}$ & $\begin{array}{c}\mathrm{r}_{e} \\
(\operatorname{arcsec})\end{array}$ & $\begin{array}{c}\mathrm{r}_{e} \\
(\mathrm{kpc})\end{array}$ & $\begin{array}{c}\mu_{e} \\
\left(\mathrm{mag} / \operatorname{arcsec}^{2}\right)\end{array}$ \\
\hline Abell 1413 & 16.63 & 3.71 & 25.4 & 57.4 & 25.0 \\
MKW 7 & 13.92 & 4.15 & 16.2 & 8.50 & 22.2 \\
\hline
\end{tabular}

Table 3. Residual model

\begin{tabular}{llllllll}
\hline \hline Cluster & $\mathrm{r}_{\text {cutoff }}$ & $\mathrm{r}_{\text {cutoff }}$ & $\beta$ & $\beta$ & $\mathrm{m}_{\text {total, v }}$ & $\begin{array}{c}\mathrm{m}_{\text {excess, v }} \\
(\mathrm{mag})\end{array}$ & $\begin{array}{c}\frac{L_{\text {excess }}}{L_{\text {total }}} \\
(\mathrm{mag})\end{array}$ \\
\hline Abell 1413 & 29.2 & 66.0 & -1.11 & -1.01 & 14.7 & 16.9 & 0.13 \\
MKW 7 & 22.6 & 11.8 & -1.24 & -0.78 & 12.6 & 14.3 & 0.21 \\
\hline
\end{tabular}


Table 4. Properties of arc candidates in Abell 1413 and MKW 7

\begin{tabular}{|c|c|c|c|c|c|c|c|c|}
\hline Name & $\alpha(2000)$ & $\delta(2000)$ & $\begin{array}{l}\text { Linear size } \\
\text { (arcsec) }\end{array}$ & $\begin{array}{l}\text { Linear size } \\
\quad(\mathrm{kpc})\end{array}$ & $\mathrm{m}_{i s o}$ & $\mathrm{M}_{i s o}$ & $\mu_{\max }$ & $\mu_{a v g}$ \\
\hline Abell 1413 Arc 1 & $11 \mathrm{~h} 55 \mathrm{~m} 17.888 \mathrm{~s}$ & $+23^{\circ} 24^{\prime} 45.70^{\prime \prime}$ & $11.3 \times 1.2^{\mathrm{a}}$ & $25.4 \times 2.8$ & $22.6 \pm 0.05^{\mathrm{b}}$ & -16.3 & 24.8 & 25.4 \\
\hline Abell 1413 Arc 2 & $11 \mathrm{~h} 55 \mathrm{~m} 20.326 \mathrm{~s}$ & $+23^{\circ} 23^{\prime} 19.94^{\prime \prime}$ & $7.0 \times 1.2^{\mathrm{a}}$ & $15.8 \times 2.8$ & $23.6 \pm 0.1$ & -15.3 & 25.1 & 25.9 \\
\hline Abell 1413 Arc 3 & $11 \mathrm{~h} 55 \mathrm{~m} 18.372 \mathrm{~s}$ & $+23^{\circ} 23^{\prime} 57.34^{\prime \prime}$ & $6.1 \times 2.0$ & $13.7 \times 4.5$ & $22.7 \pm 0.02$ & -16.2 & 25.0 & 25.4 \\
\hline Abell 1413 Arc 4 & $11 \mathrm{~h} 55 \mathrm{~m} 19.193 \mathrm{~s}$ & $+23^{\circ} 24^{\prime} 26.80^{\prime \prime}$ & $3.9 \times 1.2^{\mathrm{a}}$ & $8.8 \times 2.8$ & $23.7 \pm 0.08$ & -15.2 & 25.2 & 25.4 \\
\hline Abell 1413 Arc 5 & $11 \mathrm{~h} 55 \mathrm{~m} 19.667 \mathrm{~s}$ & $+23^{\circ} 24^{\prime} 24.51^{\prime \prime}$ & $6.0 \times 1.2^{\mathrm{a}}$ & $13.5 \times 2.8$ & $24.5 \pm 0.14$ & -14.4 & 25.5 & 26.6 \\
\hline MKW 7 Arc 1 & $14 \mathrm{~h} 33 \mathrm{~m} \mathrm{58.084s}$ & $+03^{\circ} 45^{\prime} 57.90^{\prime \prime}$ & $4.9 \times 1.8$ & $2.6 \times 1.0$ & $23.2 \pm 0.08$ & -12.1 & 25.0 & 25.8 \\
\hline
\end{tabular}

aThis dimension is unresolved in $1^{\prime \prime} .22$ seeing

b The magnitude errors are derived from SExtractor, and are an underestimate to the true errors.

Table 5. Properties of previously discovered cluster tidal debris

\begin{tabular}{|c|c|c|c|c|c|c|c|c|c|}
\hline Cluster & $\begin{array}{l}\text { Linear size } \\
(\operatorname{arcsec})\end{array}$ & $\begin{array}{l}\text { Linear size } \\
\qquad(\mathrm{kpc})^{\mathrm{a}}\end{array}$ & $\mathrm{m}$ & $\mathrm{M}^{\mathrm{a}}$ & $\mu_{\max }$ & $\mu_{a v g}$ & $\mu_{V}^{\mathrm{b}}$ & Filter & Source \\
\hline Coma TM & $180 \times 10$ & $80 \times 4$ & $\cdots$ & $\cdots$ & 26.5 & $\ldots$ & 25.6 & $\mathrm{~B}$ & TM1998 \\
\hline Coma TM & & & $\ldots$ & $\ldots$ & 25.0 & $\ldots$ & & $\mathrm{R}$ & TM1998 \\
\hline Coma LSB1 & $270 \times 60$ & $120 \times 30$ & $15.6 \pm 0.1$ & $-19.2^{\mathrm{a}} \pm 0.1$ & $\ldots$ & 25.7 & 26.3 & $\mathrm{R}$ & GW1998 \\
\hline Centaurus CR & $720 \times 10$ & $160 \times 1$ & $18.4 \pm 0.5$ & $-13.1 \pm 0.5$ & $\cdots$ & 27.8 & 26.9 & $\mathrm{~B}$ & CR2000 \\
\hline Centaurus CR & & & 16.7 & -14.8 & $\cdots$ & 26.1 & & $\mathrm{R}$ & CR2000 \\
\hline Centaurus CR & & & 16.4 & -15.11 & $\cdots$ & 25.7 & & $\mathrm{I}$ & CR2000 \\
\hline
\end{tabular}

${ }^{a}$ We assume a distance modulus of 34.83 to Coma, and 31.51 to Centaurus

${ }^{\mathrm{b}}$ Assuming $\mathrm{B}-\mathrm{V}=0.9, \mathrm{~V}-\mathrm{R}=0.6, \mathrm{~V}-\mathrm{I}=1.2($ Gregg \& West 1998$)$ 
Fig. 1. - The spectrum of the night sky at Kitt Peak National Observatory, taken from the data of Massey \& Foltz (2000). Overlaid over the spectrum is the filter transmission curve of the Washington $M$ filter used in these observations (KP1581), shown as the solid line. For comparison, a standard Harris V filter (KP1542) is also shown as the dashed line. The Washington $\mathrm{M}$ filter contains fewer strong sky-emission lines compared to the $\mathrm{V}$ filter, most notably O I $\lambda 5577$.

Fig. 2.- A comparison of T2KA photometry obtained from a 60s test exposure and a median-combined 900s exposure of MKW 7. Although there is large scatter (primarily from photon noise in the 60s exposure), there is a clear systematic residual with instrumental magnitude, identical to that seen by Mochesjka et al. (2001). The line denotes the leastsquares linear fit through the data.

Fig. 3.- The difference between surface brightness of data corrected for the non-linearity effect found by Mochesjka et al. (2001) for the T2KA detector, and un-corrected data, over the range of surface brightnesses applicable to our survey. Note that the effect is nowhere greater than 0.012 magnitudes, and the effect is relatively constant over the range of surface brightnesses that ICL would be present $\left(\mu_{V}=24-30\right)$

Fig. 4.- Images of the 20 blank sky frames used in constructing the master sky flat, after being flat-fielded and averaged into 50 pixel bins. The small scale variations are due to bright stars and galaxies in each individual frame, but the large scale variations are due to changes in the sky illumination. These large-scale features are partially removed by the plane normalization. The grid-like scattered light pattern can also be barely seen in three of the frames (bottom row, first and fourth column, and second row from bottom, fifth column). These grid-like patterns are almost invisible in the un-binned images.

Fig. 5.- Our final, median-combined image for Abell 1413. North is at the left of this image, and east is at the bottom. The image is 10 ' 2 square, corresponding to a linear distance of 1.5 Mpc at our adopted distance to Abell 1413. Note the incredible richness of this cluster: almost every bright object in the frame with the exception of the two saturated stars at the bottom is a galaxy.

Fig. 6.- Our final, median-combining image for MKW 7. North is at the left of this image, and east is at the bottom. The image is 10 ' 2 square, corresponding to a linear distance of $330 \mathrm{kpc}$ at our adopted distance to MKW 7. The bright, saturated star superimposed near MKW7s nucleus cannot be seen in this image greyscale. 
Fig. 7.- The surface brightness profile of a saturated star on our Abell 1413 image, averaged azimuthally. As can be clearly seen, the profile extends to very large radii. The solid horizontal line is set at the surface brightness limit of $1 \mathrm{ADU}_{\text {pixel }}^{-1}$ above the sky value, corresponding to a surface brightness of 28.48 mag $\operatorname{arsec}^{-2}$.

Fig. 8. - The brightness distribution of all objects in the Abell 1413 (left) and MKW 7 (right) fields found by SExtractor that have a stellarity index less than 0.5, shown as the filled dots. Note that this is not the cluster luminosity function, as no background subtraction has been done, and the blending parameter of SExtractor has been turned off. The brightness distribution is compared to the brightness distribution of negative noise spikes, shown as the open diamonds. As can be clearly seen, the brightness distribution steepens at the same magnitude $(\mathrm{m} \approx 23)$ that the negative noise spikes become present in great numbers. This denotes where the overmasking problem begins.

Fig. 9.- A region of MKW 7 shown through all stages of the masking process. From left to right, and top to bottom the sub-images are: 1) The original image, 2) the image multiplied by the stellar mask found through DAOFIND 3) the previous image multiplied by the mask from SExtractor, 4) the previous mask, with the "over-masking" correction applied, 5) the previous image, with the surrounding pixels correction made, and 6) the previous image after manual masking. The grey-scale in all of the images is 10 ADU above and below the sky value, and the standard deviation of the sky background is 5.8 ADU per pixel. The masking procedure removes the vast majority of stellar and galaxy light in the image.

Fig. 10. - The binned-up image of Abell 1413, with a greyscale stretch of five ADU above and below the sky value. North is again to the left, and east is at the bottom of this image. As the bins increase in radius from the central cD galaxy, their fluxes systematically change, as discussed in the text. At the far right of this image, a large-scale flat-fielding error of 1 ADU is clearly visible.

Fig. 11. - The binned-up image of MKW 7, similar to that of Figure 10. In this case, no large-scale flat-fielding error is present.

Fig. 12.- The histogram of sky values for Abell 1413 (left) and MKW 7 (right), binned up into 0.25 ADU intervals. See the text for the description of how this histogram was created. Ideally, the sky values should all equal zero, but due to large-scale flat-fielding errors, and the wings of unmasked stars and galaxies, there is a dispersion about zero. We estimate the error in the sky to be \pm 1 ADU. 
Fig. 13.- The radial surface brightness profiles of Abell 1413 (left) and MKW 7 (right), as measured by our data. The solid horizontal solid line at $\mu \sim 26.5$ and the dashed line at $\mu \sim 28.3$ indicate where our data has a signal-to-noise of five and one, respectively. The vertical dot-dashed line indicates the radial scale of the seeing disk in these units. Note that the effects of seeing extend over several seeing radii. 
Fig. 14. - The radial surface brightness profile of MKW 7, as measured by our data (filled circles), compared to the $B$ band photographic surface photometry of Morbey \& Morris (1983) (open diamonds). The $B$ data has been shifted by 1.1 magnitudes vertically to account for passband differences. The dashed line at $\mu=26.5$ indicates where our data reaches a signal-to-noise of five and the solid line at $\mu=28.3$ indicates where our data has a signal-to-noise of one. The two profiles agree to with \pm 0.1 magnitudes except at large radii.

Fig. 15. - The radial surface brightness profile of Abell 1413, as measured by our data (filled circles), compared to the $V$ band photographic surface photometry of Schombert (1986) (open diamonds). The dashed line at $\mu=26.5$ indicates where our data reaches a signal-tonoise of five and the solid line at $\mu=28.3$ indicates where our data has a signal-to-noise of one. The two profiles disagree at large and small radii. See the text for discussion.

Fig. 16. - An expanded view of the surface brightness profile of Abell 1413 at small radii. As before, the filled circles are our data, but the open diamonds are now the $r$ band CCD data of Schneider, Gunn \& Hoessel (1983), after being displaced by 0.3 magnitudes. The two profiles are in good agreement.

Fig. 17.- A expanded view of the surface brightness profile of Abell 1413 at large radii. As before, the filled circles are our data, the open diamonds are the data of Schombert (1986), and the two horizontal lines show signal-to-noise ratios of five, and one respectively. The filled squares indicate the effect of overestimating the sky by 1 ADU in our data.

Fig. 18. - The residuals in our surface brightness profile for Abell 1413 (left) and MKW 7 (right), after the best-fitting de Vaucouleurs law of the inner regions has been subtracted. Negative residuals indicate a magnitude excess, and positive residuals indicate a flux deficit. The best-fitting excess model (see the text), is plotted as the solid line. There is a clear deviation above the de Vaucouleurs law in both cases. Such behavior for brightest cluster members in poor clusters is unexpected (Thuan \& Romanishin 1981).

Fig. 19.- A plot of the $\mathrm{A}_{3}, \mathrm{~A}_{4}, \mathrm{~B}_{3}$, and $\mathrm{B}_{4}$ terms for Abell 1413 (left) and MKW 7 (right), as a function of radius. Clear non-zero terms are present in both data sets, indicating that there are non-elliptical residuals present in the data. 
Fig. 20.- Our residual image for Abell 1413, after the best-fitting elliptical model of the cD + ICL has been subtracted. The black ellipse shows where the measured surface brightness has a signal-to-noise greater than five. There is an indication of a low surface brightness bridge between two luminous galaxies located up and to the right of the $\mathrm{cD}$ nucleus (shown by the square), but the results are not conclusive. No obvious large-scale tidal features are apparent, but there are a number of small arc-like structures clearly visible. See the text for discussion of these arcs.

Fig. 21.- Our residual image for MKW 7, after the best-fitting elliptical model of the cD + ICL has been subtracted. The black ellipse shows where the measured surface brightness has a signal-to-noise greater than five. A large tidal plume is apparent leading from the center of the image to the right (south), and up (west) of the galaxy's nucleus.

Fig. 22.- The best-fitting values for the position angle and ellipticity of our ELLIPSE fits, as a function of radius for Abell 1413 (top) and MKW 7 (bottom).

Fig. 23. - An 2' $7 \times 22^{\prime} 7$ image of the central region of Abell 1413, with the cD galaxy subtracted, and point sources masked out. North is to the left, and east is at the bottom of this figure. Two arc-like structures are clearly visible $(1,2)$, with another three (3-5) possible. Note that arcs 1-3 lie tangentially to the $\mathrm{cD}$ galaxy, implying that these features might be due to gravitational lensing.

Fig. 24.- An 1.' $4 \times 1$.' 4 image of the central region of MKW 7, with the cD galaxy subtracted. North is to the left, and east is at the bottom of this figure. There is one arc-like feature clearly seen away from the galaxy nucleus. 
This figure "f1.gif" is available in "gif" format from: http://arxiv.org/ps/astro-ph/0204467v1 
This figure "f2.gif" is available in "gif" format from: http://arxiv.org/ps/astro-ph/0204467v1 
This figure "f3.gif" is available in "gif" format from: http://arxiv.org/ps/astro-ph/0204467v1 
This figure "f4.jpg" is available in "jpg" format from: http://arxiv.org/ps/astro-ph/0204467v1 
This figure "f5.jpg" is available in "jpg" format from: http://arxiv.org/ps/astro-ph/0204467v1 
This figure "f6.jpg" is available in "jpg" format from: http://arxiv.org/ps/astro-ph/0204467v1 
This figure "f7.gif" is available in "gif" format from: http://arxiv.org/ps/astro-ph/0204467v1 
This figure "f8a.gif" is available in "gif" format from: http://arxiv.org/ps/astro-ph/0204467v1 
This figure "f8b.gif" is available in "gif" format from: http://arxiv.org/ps/astro-ph/0204467v1 
This figure "f9.jpg" is available in "jpg" format from: http://arxiv.org/ps/astro-ph/0204467v1 
This figure "f10.jpg" is available in "jpg" format from: http://arxiv.org/ps/astro-ph/0204467v1 
This figure "f11.jpg" is available in "jpg" format from: http://arxiv.org/ps/astro-ph/0204467v1 
This figure "f12a.gif" is available in "gif" format from: http://arxiv.org/ps/astro-ph/0204467v1 
This figure "f12b.gif" is available in "gif" format from: http://arxiv.org/ps/astro-ph/0204467v1 
This figure "f13a.gif" is available in "gif" format from: http://arxiv.org/ps/astro-ph/0204467v1 
This figure "f13b.gif" is available in "gif" format from: http://arxiv.org/ps/astro-ph/0204467v1 
This figure "f14.gif" is available in "gif" format from: http://arxiv.org/ps/astro-ph/0204467v1 
This figure "f15.gif" is available in "gif" format from: http://arxiv.org/ps/astro-ph/0204467v1 
This figure "f16.gif" is available in "gif" format from: http://arxiv.org/ps/astro-ph/0204467v1 
This figure "f17.gif" is available in "gif" format from: http://arxiv.org/ps/astro-ph/0204467v1 
This figure "f18a.gif" is available in "gif" format from: http://arxiv.org/ps/astro-ph/0204467v1 
This figure "f18b.gif" is available in "gif" format from: http://arxiv.org/ps/astro-ph/0204467v1 
This figure "f19.gif" is available in "gif" format from: http://arxiv.org/ps/astro-ph/0204467v1 
This figure "f20.jpg" is available in "jpg" format from: http://arxiv.org/ps/astro-ph/0204467v1 
This figure "f21.jpg" is available in "jpg" format from: http://arxiv.org/ps/astro-ph/0204467v1 
This figure "f22.gif" is available in "gif" format from: http://arxiv.org/ps/astro-ph/0204467v1 
This figure "f23.jpg" is available in "jpg" format from: http://arxiv.org/ps/astro-ph/0204467v1 
This figure "f24.jpg" is available in "jpg" format from: http://arxiv.org/ps/astro-ph/0204467v1 